\title{
Application of 3D Ultrasound in Gynecology
}

\author{
Kazunori Baba
}

Correspondence: Center for Maternal, Fetal and Neonatal Medicine, Saitama Medical Center

Saitama Medical School, 1981 Kamoda, Kawagoe, Saitama 350-8558, Japan

E-mail: baba-tokyo@umin.ac.jp

\section{UTERUS}

Two-dimensional transvaginal ultrasound can demonstrate sagittal and transverse sections of the uterus clearly. However it can hardly depict a coronal section of the uterus which is the best section to show abnormalities of the uterine cavity. Threedimensional ultrasound can depict any arbitrary section including a coronal section of the uterus easily (Fig. 1). However, the whole uterine cavity (the endometrium) is hardly depicted by a single coronal section when the uterine cavity is curved too much (Fig. 2).

Three-dimensional images can depict the whole uterine cavity even in such cases. Furthermore, the contrast between the endometrium and the myometrium is higher on a 3D image than on a sectional image (Fig. 3). A 3D image of the uterine cavity by maximum intensity projection (maximum mode) is obtained by simply setting ROI (region of interest) to surround the endometrium when it is hyperechoic in the secretory phase (Fig. 4). A 3D image of the uterine cavity by surface mode is obtained by setting ROI along the endometrium (Fig. 5). Volume imaging (quasi-thick-slice-width imaging or volume contrast imaging) is also applicable for depicting a coronal view of the uterus (Fig. 6) when the uterine cavity is not curved too much. Figure 7 shows the difference of 3D images with different modes.

A coronal view of the uterus by 3D ultrasound demonstrates various congenital uterine abnormalities luminously (Fig. 8). Conventional transvaginal 2D ultrasound can hardly depict the

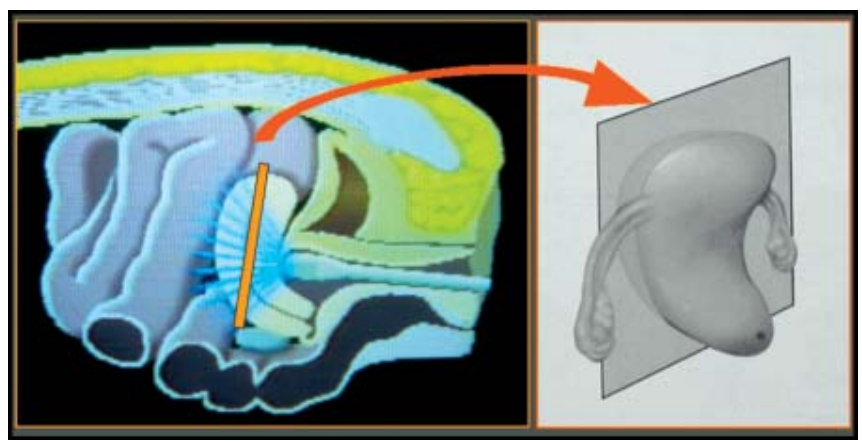

Fig. 1: Three-dimensional transvaginal ultrasound can depict a coronal section of the uterus

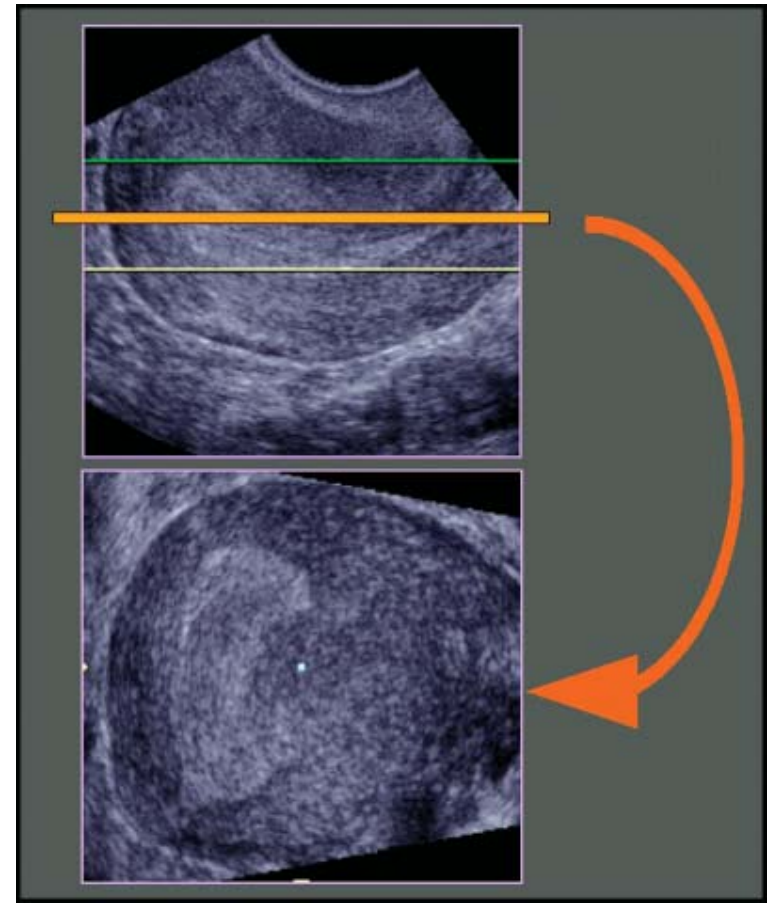

Fig. 2: A single coronal section of the uterus cannot demonstrate the whole uterine cavity (the endometrium) when the uterine cavity curved too much. (Upper) A sagittal section of the uterus. (Lower) A coronal section of the uterus

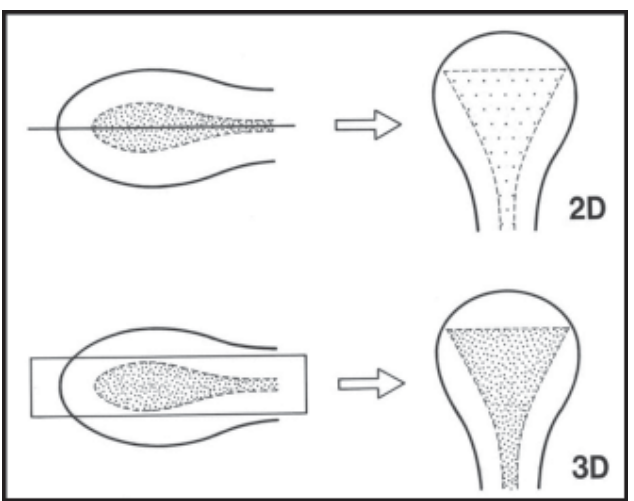

Fig. 3: Higher contrast is obtained by using $3 \mathrm{D}$ data than by using only one sectional data 


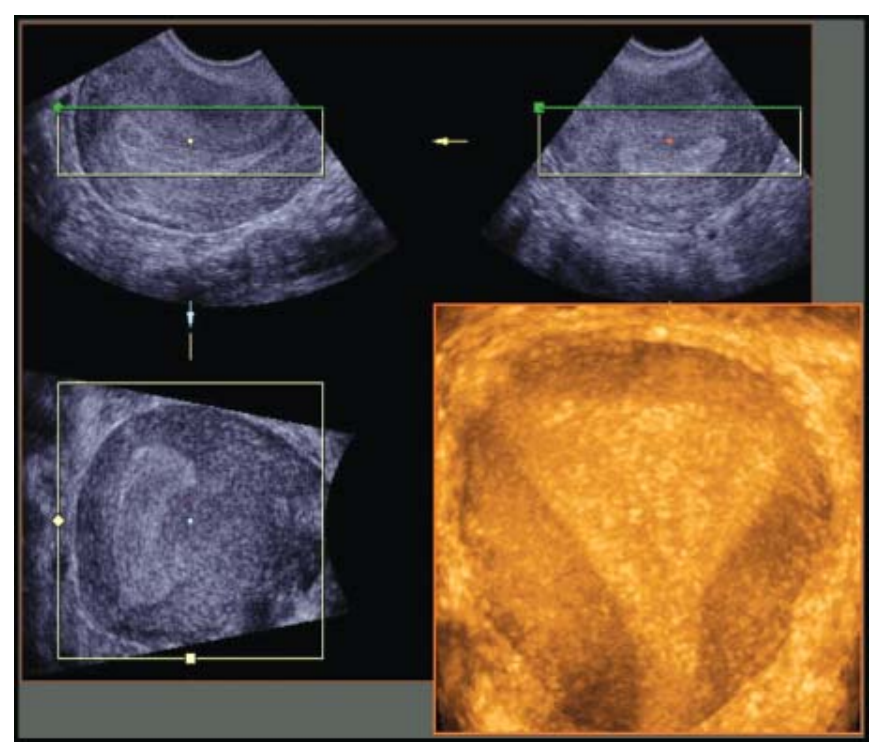

Fig. 4: A coronal view of the uterus by maximum mode (Lower right)

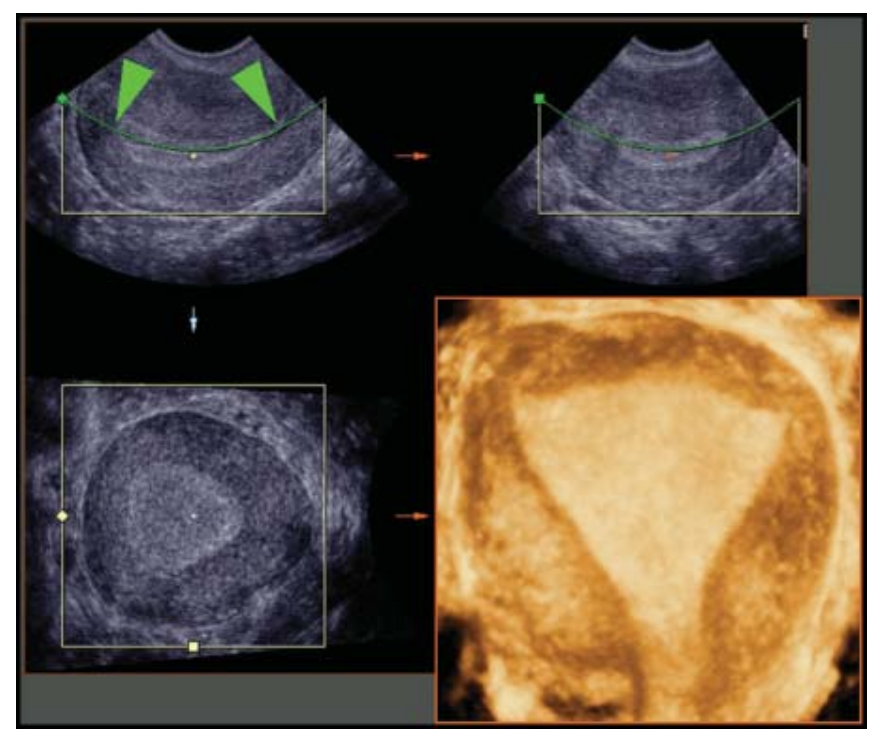

Fig. 5: A coronal view of the uterus by surface mode (Lower right). Note that ROI (arrow heads) is adjusted to the curve of the endometrium (Upper left)

whole image of an intrauterine contraceptive device in the uterine cavity, while 3D ultrasound can show it clearly (Fig. 9). Transvaginal 3D ultrasound demonstrates even a microinsert contraceptive coil within the proximal fallopian tube. ${ }^{1}$

A coronal view shows exactly where a gestational sac is (Fig. 10). A gestational sac out of the endometrial cavity is clearly seen in a case of interstitial pregnancy (Fig. 11).

Three-dimensional ultrasound shows the tip of an embryo transfer catheter and the location where embryos were

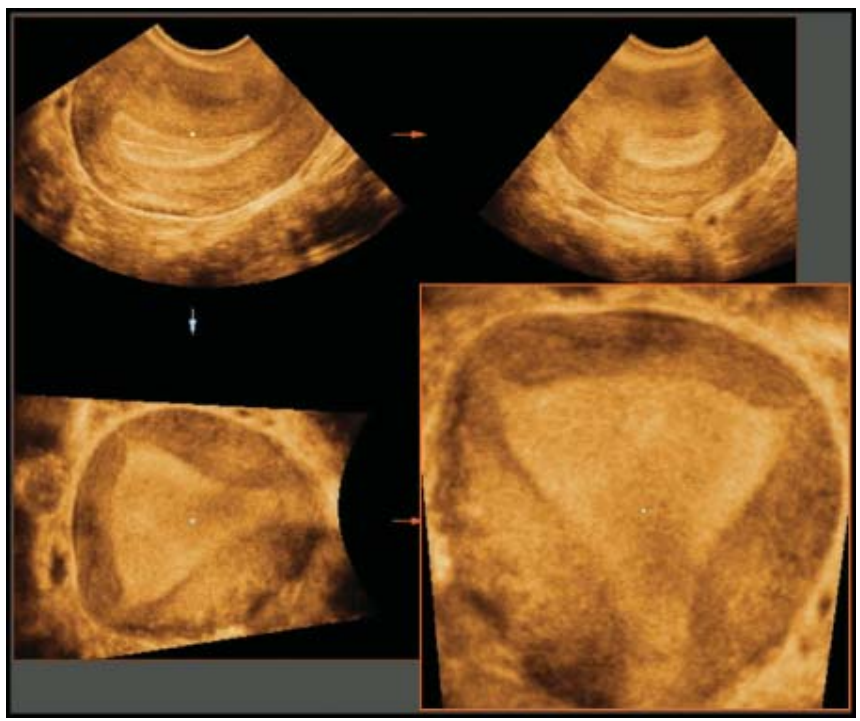

Fig. 6: A coronal view of the uterus by volume imaging (Lower left and right). (Lower right) The original image (Lower left) is enlarged and rotated

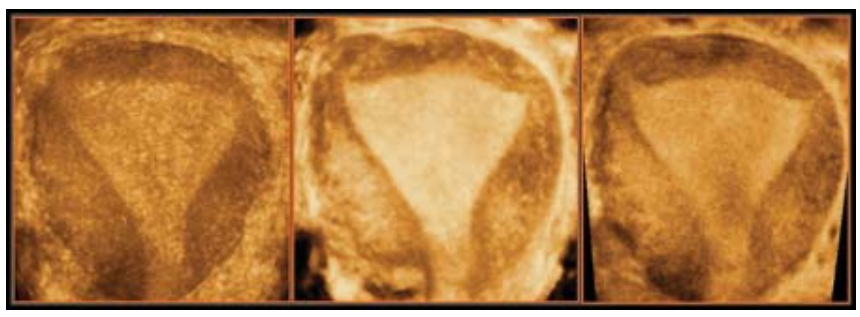

Fig. 7: Comparison of images by different modes. From left to right, maximum mode, surface mode and volume imaging. Note that bilateral edges of the uterine cavity are depicted clearly by surface mode and volume imaging. Partes uterina tubae are depicted by volume imaging

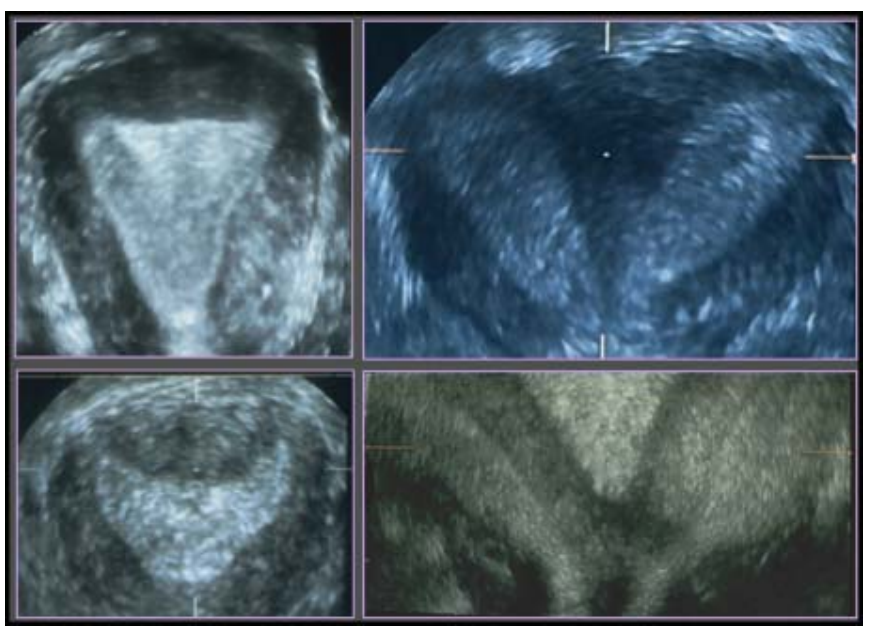

Fig. 8: Coronal views of uteri. (Upper left) Normal uterus. (Lower left) Arcuate uterus. (Upper right) Septate uterus. (Lower right) Bicornis uterus 


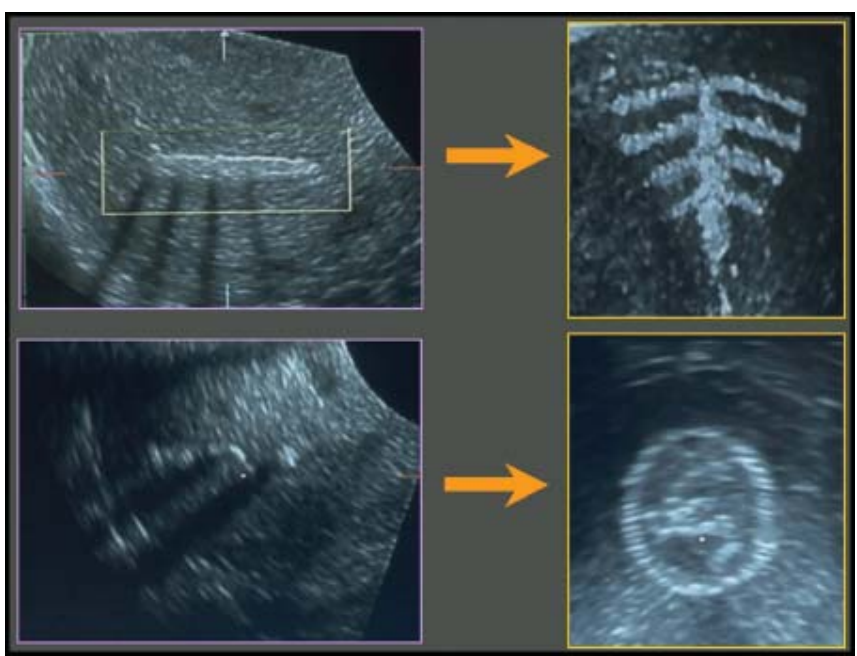

Fig. 9: Intrauterine contraceptive devices in the uterus. (Left) Sagittal sections of uteri. (Right) Coronal views of uteri

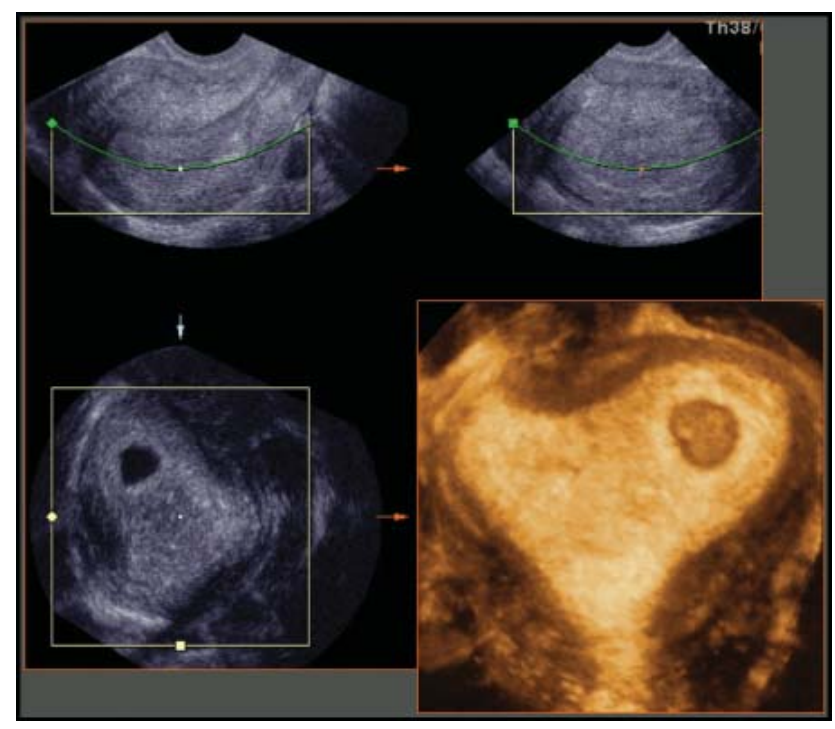

Fig. 10: A coronal view of the uterus demonstrates normal location of a gestational sac luminously (Lower right)

transferred (Fig. 12). Preliminary data showed that about $80 \%$ of embryos implanted in the same area to which they had been transferred. ${ }^{2}$

Sonohysterography makes abnormalities in and of the uterine cavity clear. Three-dimensional sonohysterography shows adhesion of the uterine cavity clearly (Fig. 13). An endometrial polyp can be analyzed minutely by translating and rotating each image of three orthogonal planes (Fig. 14). A 3D image of the polyp can be obtained by removing unnecessary part of the uterine wall (Fig. 15).

Inside of a cystic degenerated leiomyoma can be demonstrated three-dimensionally (Fig. 16A). A fold of the wall is clearly seen by rotating the 3D image (Fig. 16B).

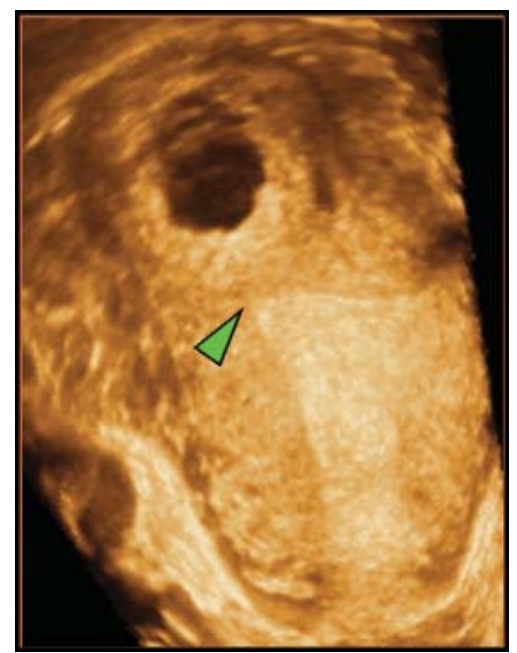

Fig. 11: Interstitial pregnancy. A gestational sac is seen out of an edge (arrow head) of the uterine cavity

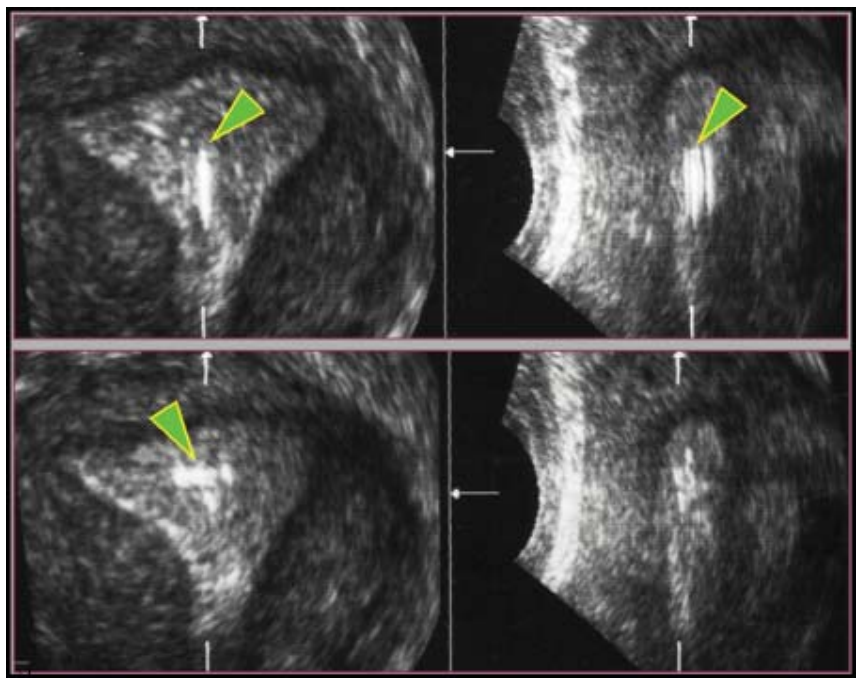

Fig. 12: Three-dimensional ultrasound in embryo transfer (ET). (Upper) The tip of an ET catheter (arrow head) can be seen on a coronal section (Left) and a sagittal section (Right). (Lower) Immediately after ET. Air bubbles (arrow head) indicate the location where the embryos were transferred

\section{Color/Power Doppler}

Abnormal blood flows in the uterus are demonstrated by Doppler ultrasound (Fig. 17A). Three-dimensional power Doppler images (Fig. 17B) demonstrate the vascular structure more clearly than 2D images. A 3D power Doppler image shows feeding vessels to an endometrial polyp and its origin luminously (Fig. 18).

A part of an abnormal blood flow of an arteriovenous (AV) shunt in the myometrium is depicted by 2D Doppler ultrasound (Fig. 19A). A three-dimensional Doppler image demonstrates the whole structure of abnormal blood flow and vessels of both inflow and outflow (Figs 19B and 20). 


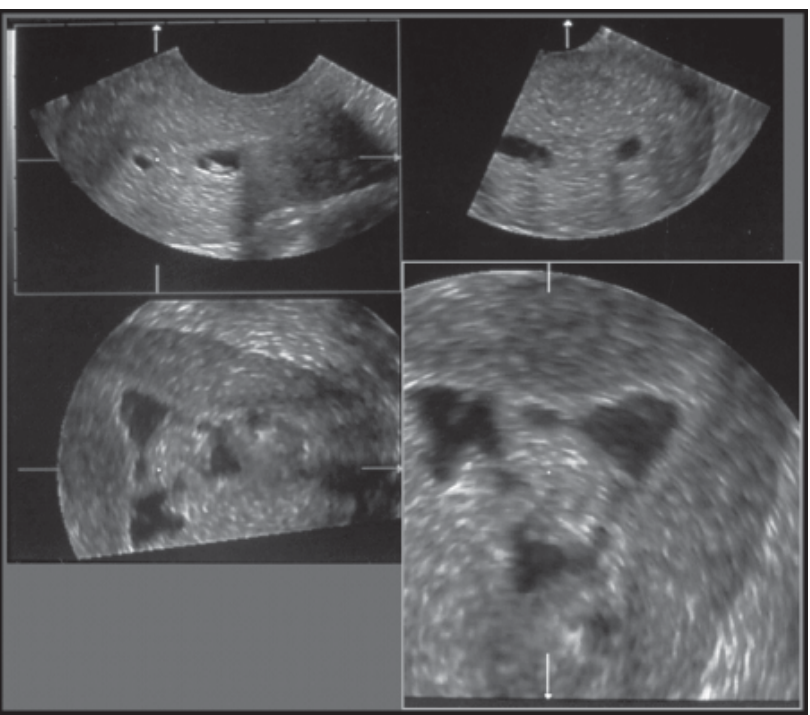

Fig. 13: Three-dimensional sonohysterography. A coronal section of the uterus with Asherman's syndrome (Lower right) ${ }^{3}$

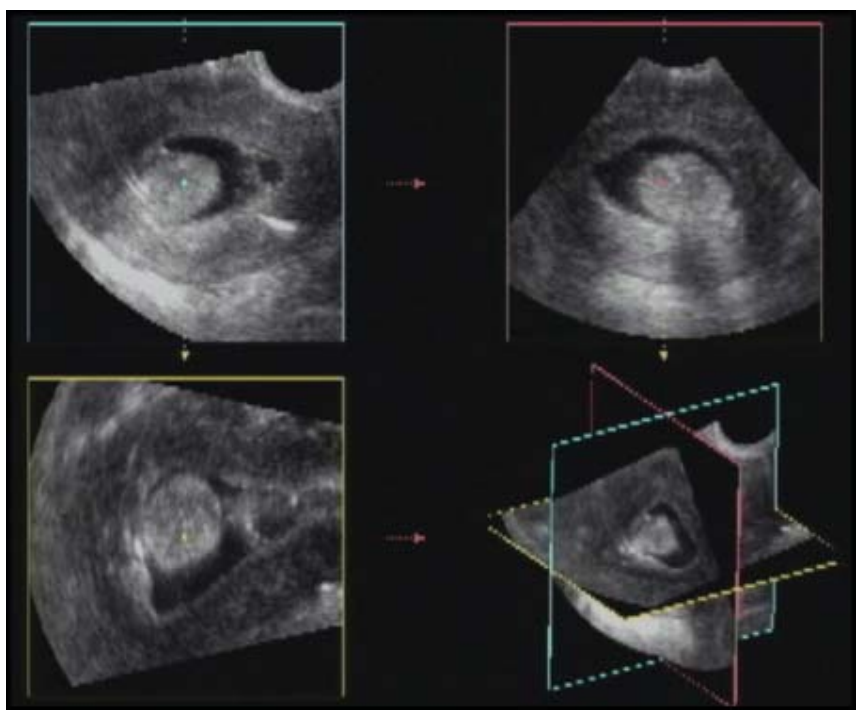

Fig. 14: Three-dimensional sonohysterography. A three-orthogonal-plane of an endometrial polyp

\section{Volume Measurement}

The endometrial cavity or the endometrium can be extracted on a three-orthogonal-plane manually and its volume can be measured accurately (Figs 21 A and B). Volume measurement of the endometrium may be useful for evaluating hormonal effects to the endometrium and hyperplasia of the endometrium.

\section{OVARY AND TUBE}

All follicular cysts can be demonstrated on a 3D image by minimum intensity projection (minimum mode) in a polycystic ovary (Fig. 22A). Each cyst is demonstrated more sharply by

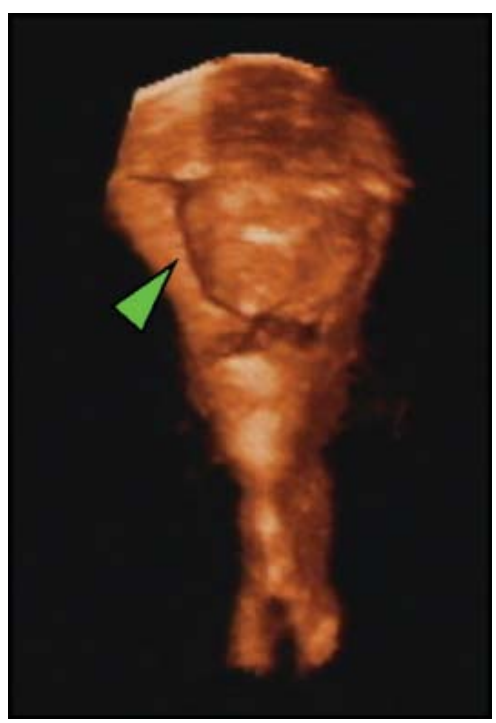

Fig. 15: A 3D image of the endometrial polyp (arrow head). Frontal and lateral uterine walls were removed electrically
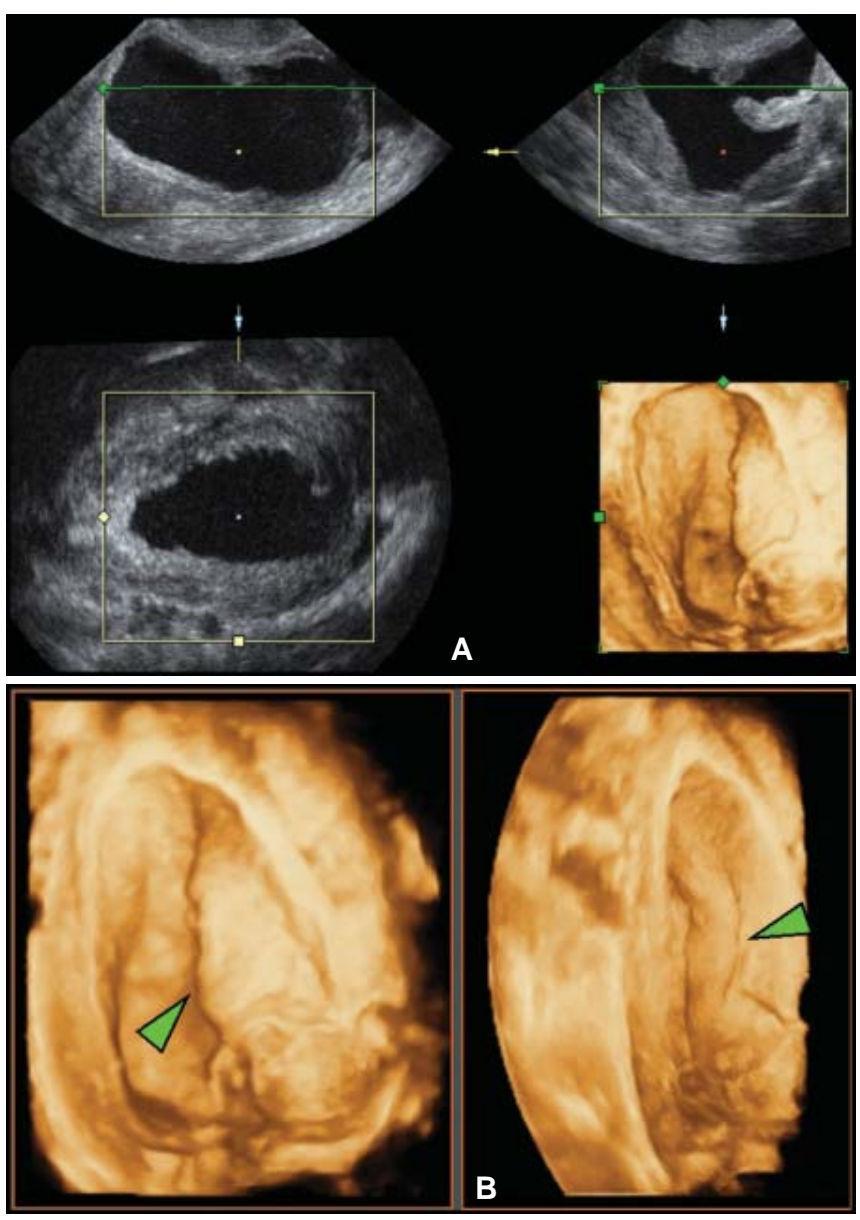

Figs 16A and B: A cystic degenerated leiomyoma of an 85-year-old woman. Liquid in the leiomyoma was aspirated partially. (A) ROI setting. (B) 3D inside views of the leiomyoma with a fold of the wall (arrow head) 

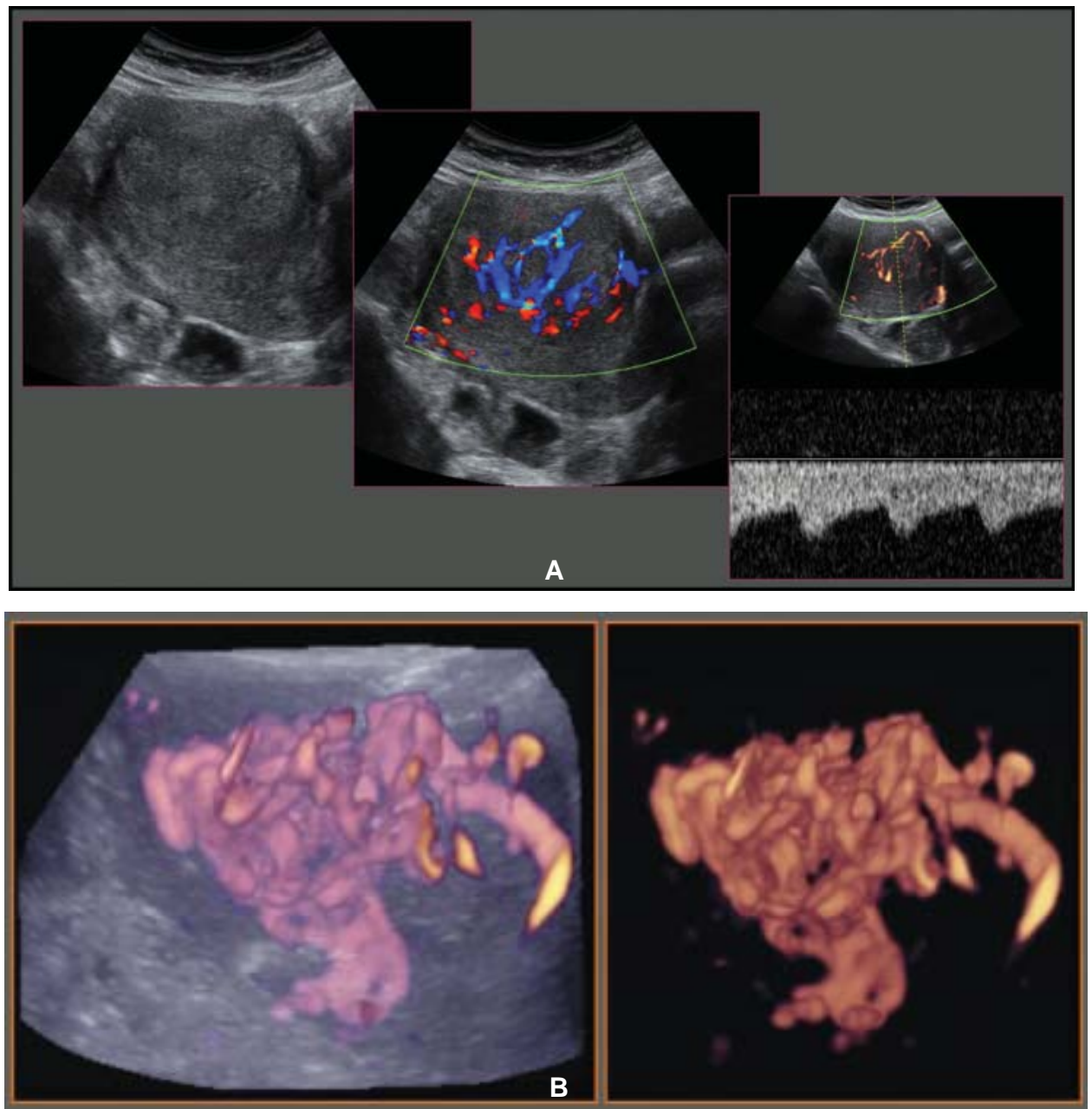

Figs 17A and B: Endometrial cancer. (A) Two-dimensional images of the uterus with and without Doppler. (B) Three-dimensional power Doppler images show rich and irregular neovascularity

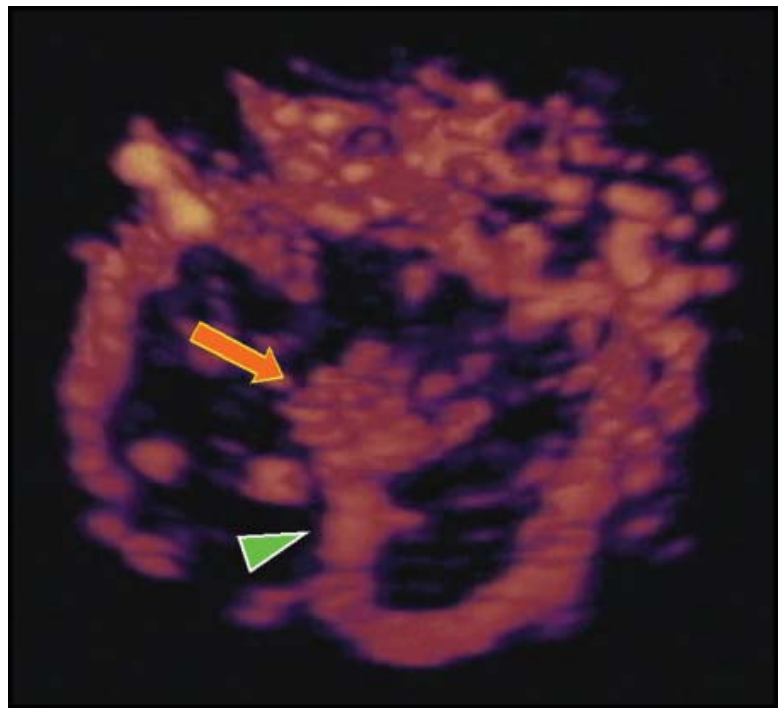

Fig. 18: A 3D power Doppler image of the uterus. Blood flows in an endometrial polyp (arrow) and feeding vessels (arrow head) are seen 

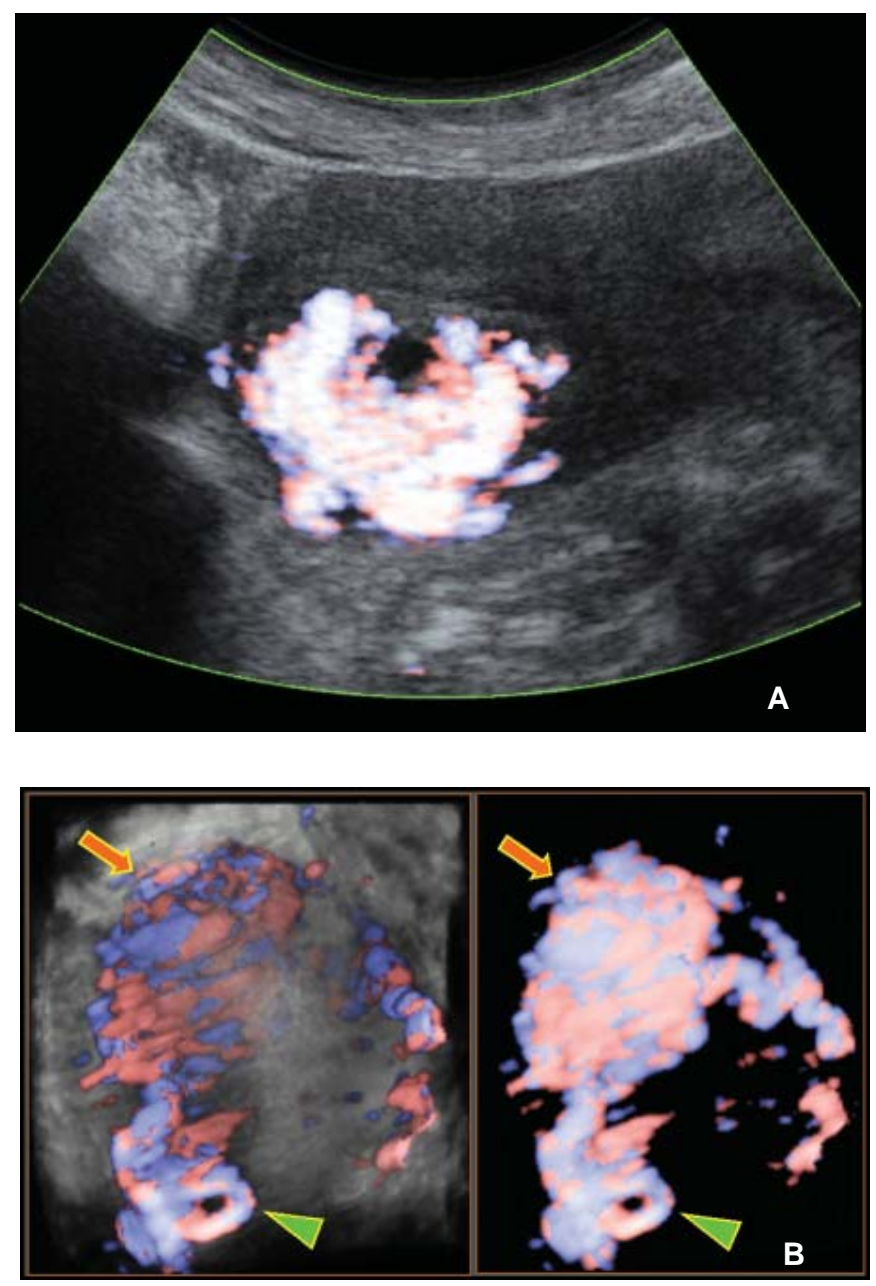

Figs 19A and B: Doppler images of an AV shunt found after D \& C. (A) A 2D image. (B) Three-dimensional images show both an AV shunt (arrow) and vessels (arrow head)

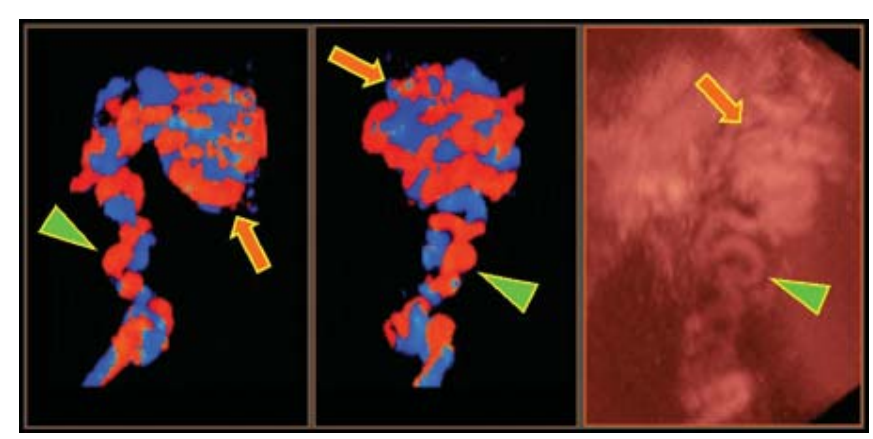

Fig. 20: Three-dimensional images of another AV shunt found after $D$ \& C. (Left and middle) Color Doppler images demonstrate AV shunt (arrow) and dilated vessels (arrow head). (Right) A B-flow 3D image
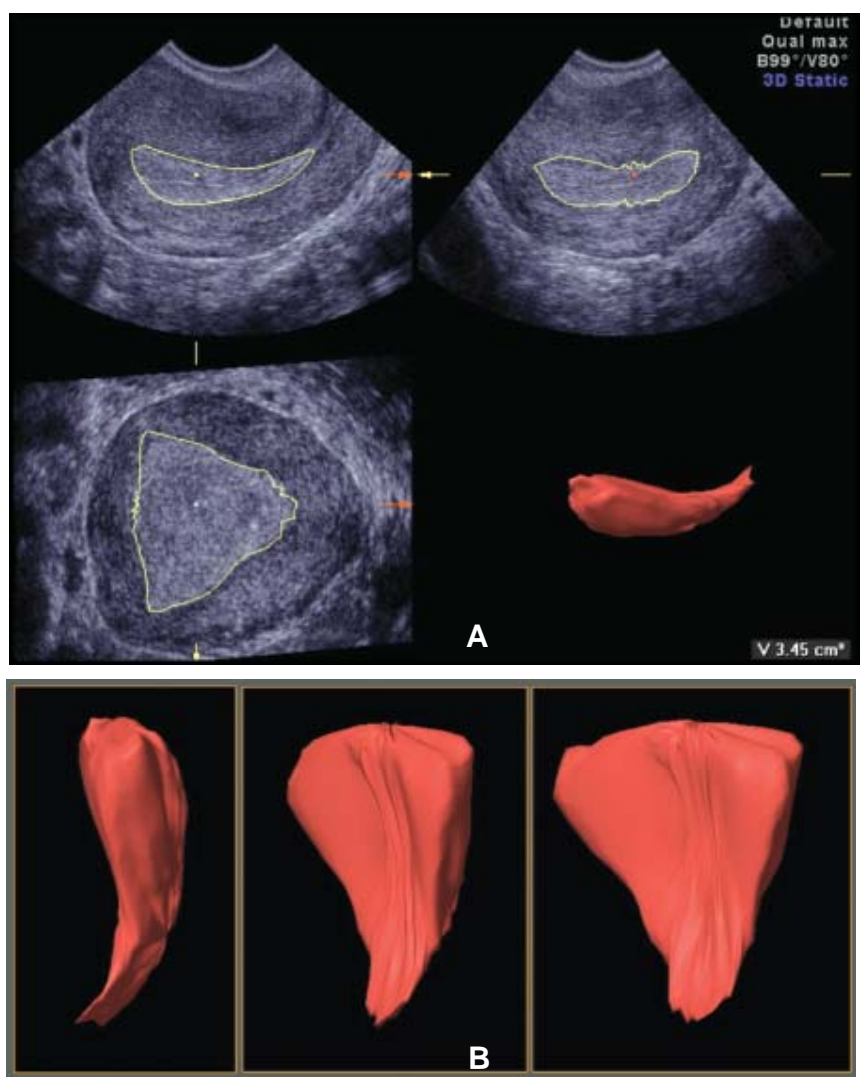

Figs 21A and B: Three-dimensional images of the endometrial cavity or the endometrium. (A) Extraction of the endometrial cavity and volume measurement. (B) Three-dimensional images of the endometrial cavity

inverting black and white (inversion mode) and by removing unnecessary surrounding structures (Fig. 22B).

An image by 2D ultrasound depicts only one section of an ovarian cyst and small solid parts on the cyst's wall tend to be overlooked. Three-dimensional ultrasound shows the inner side of the wall three-dimensionally by setting ROI properly (Fig. 23A) and solid parts can be seen luminously (Fig. 23B).

A large hydrosalpinx may be made a wrong diagnosis as an ovarian cyst (Fig. 24A). Even a three-orthogonal-plane and a surface rendered 3D image demonstrate it like an ovarian cyst (Fig. 24B). Three-dimensional images by minimum mode (Fig. 24C) and inversion mode (Fig. 24D) demonstrate its tubular structure and lead to the proper diagnosis.

\section{Color/Power Doppler}

A corpus luteum is surrounded by a net of blood vessels (Fig. 25). A 3D Doppler image can demonstrate more than one corpus luteum clearly by showing the nets of blood vessels (Fig. 26). 

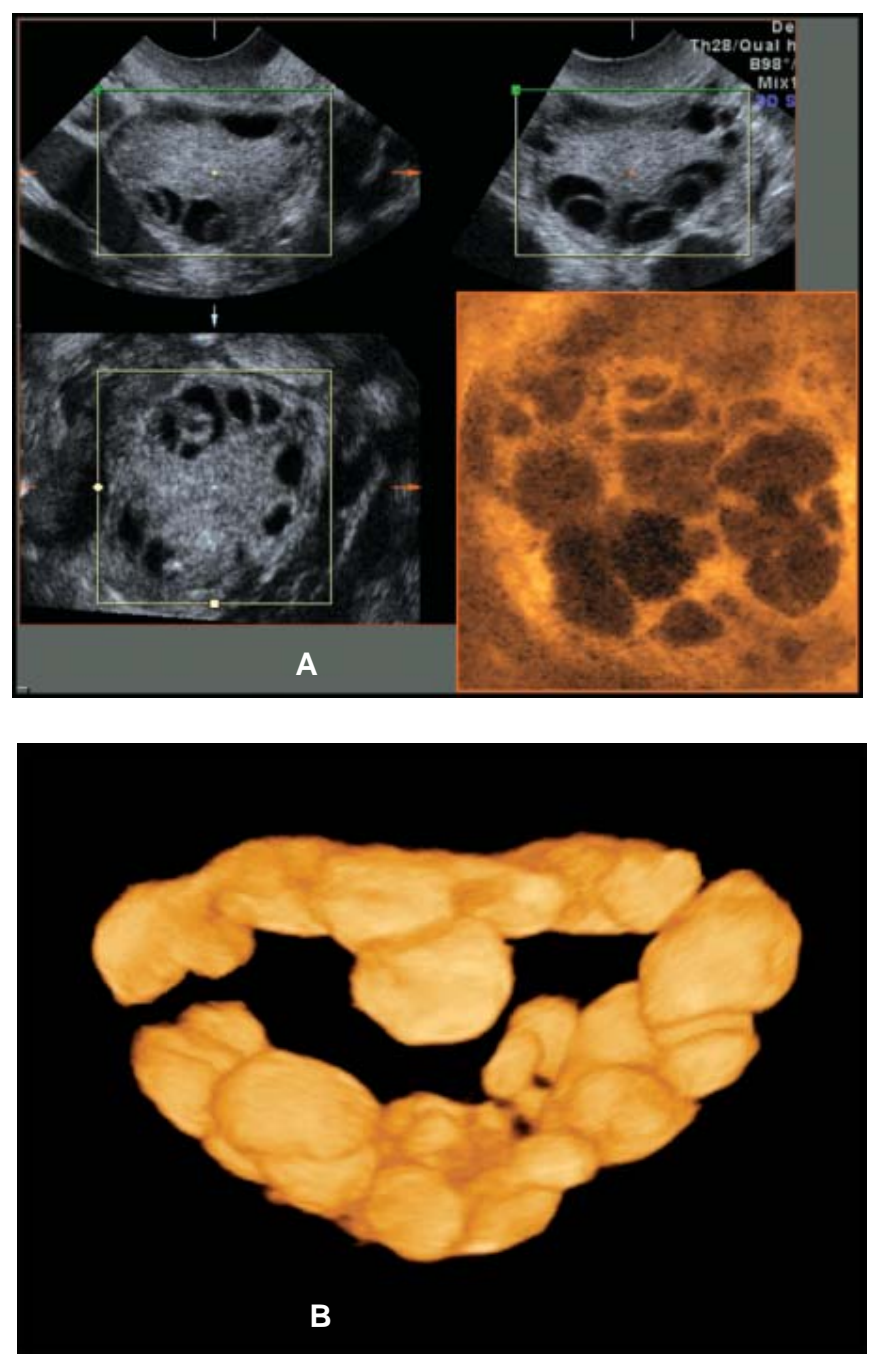

Figs 22A and B: Three-dimensional images of a polycystic ovary. (A) A 3D image by minimum mode (Lower right). (B) A 3D image of a bunch of cysts by inversion mode
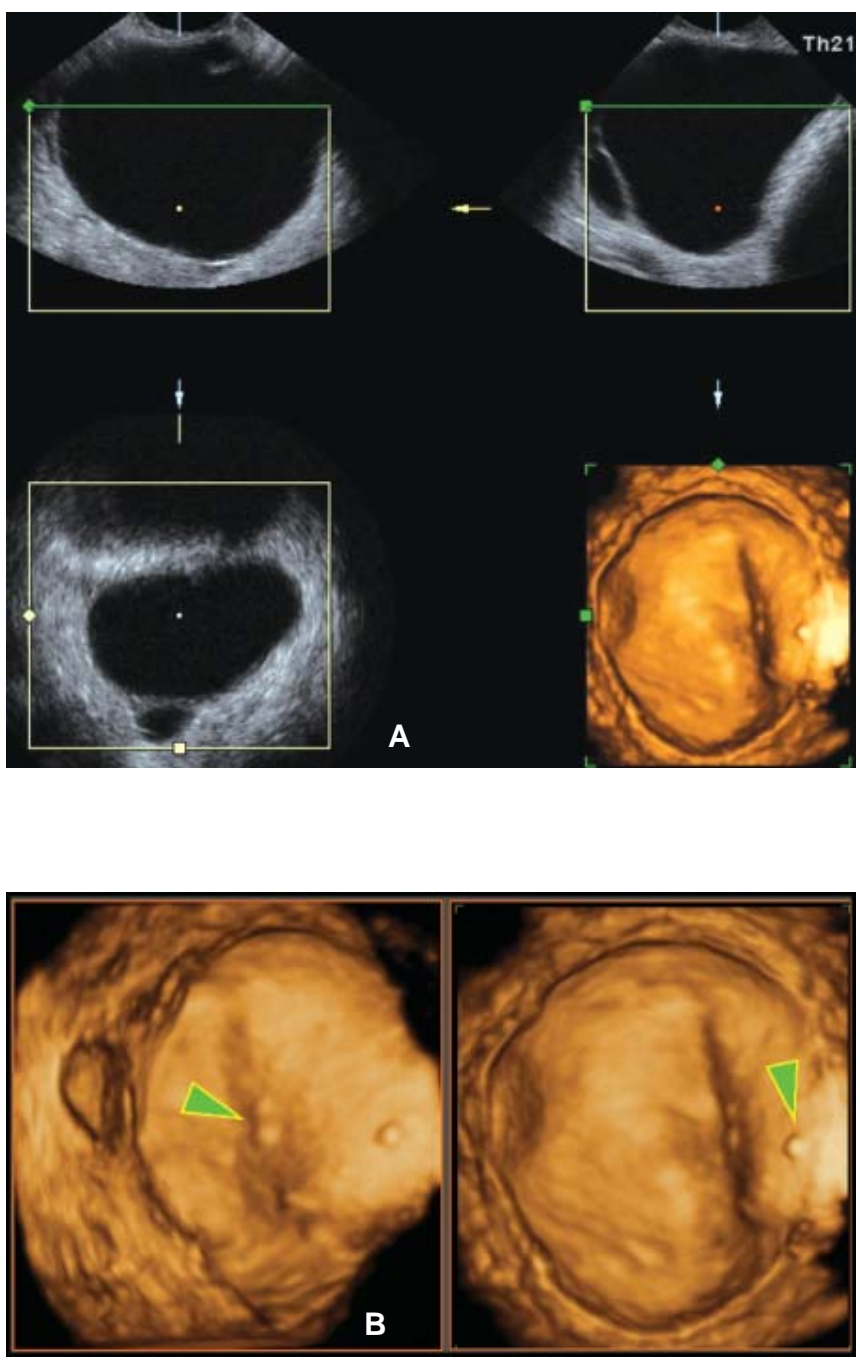

Figs 23A and B: Three-dimensional images of an ovarian cyst. (A) $\mathrm{ROI}$ setting for surface mode. Note that any of three sectional images does not depict any solid part. (B) Three-dimensional images. Solid parts on the wall (arrow head) are clearly seen
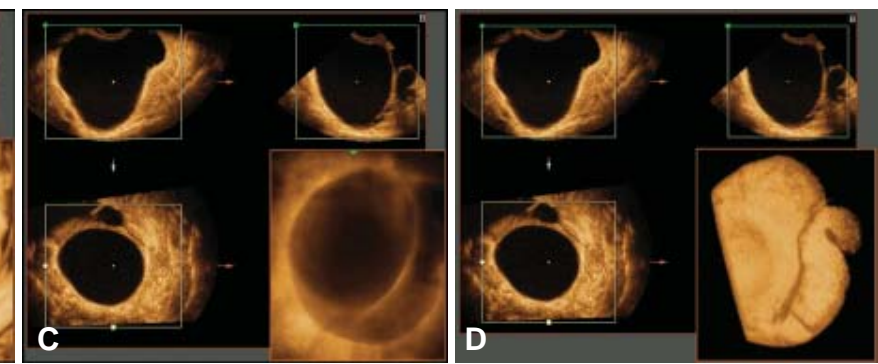

Fig. 24: Hydrosalpinx. (A) A tomographic image. (B) A 3D image by surface mode (lower right). (C) A 3D image by minimum mode and X-ray mode (Lower right). (D) A 3D image by inversion mode (Lower right) 


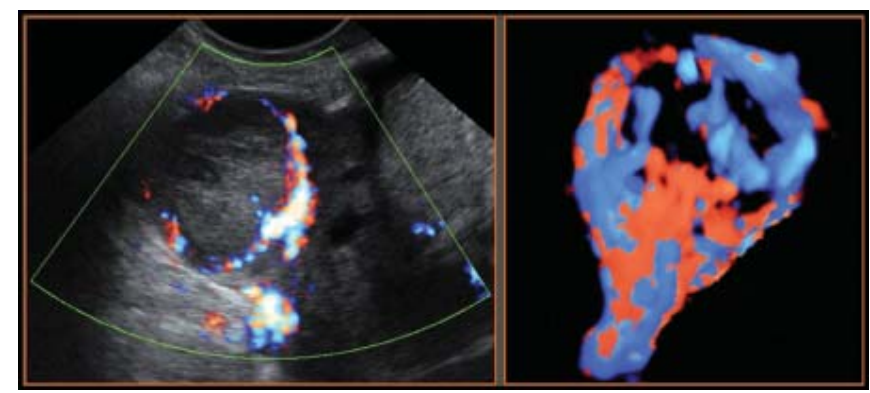

Fig. 25: Doppler images of vessels around a corpus luteum. (Left) A 2D image. (Right) A 3D image

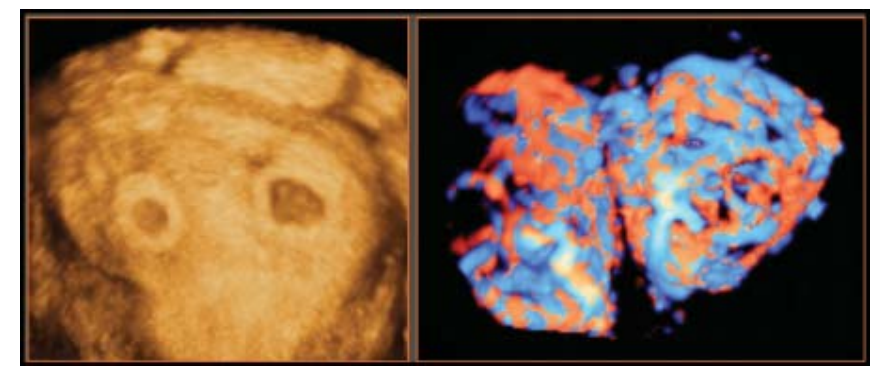

Fig. 26: DD twin pregnancy. (Left) Two gestational sacs. (Right) A Doppler 3D image shows two vascular nets indicating two corpora luteum

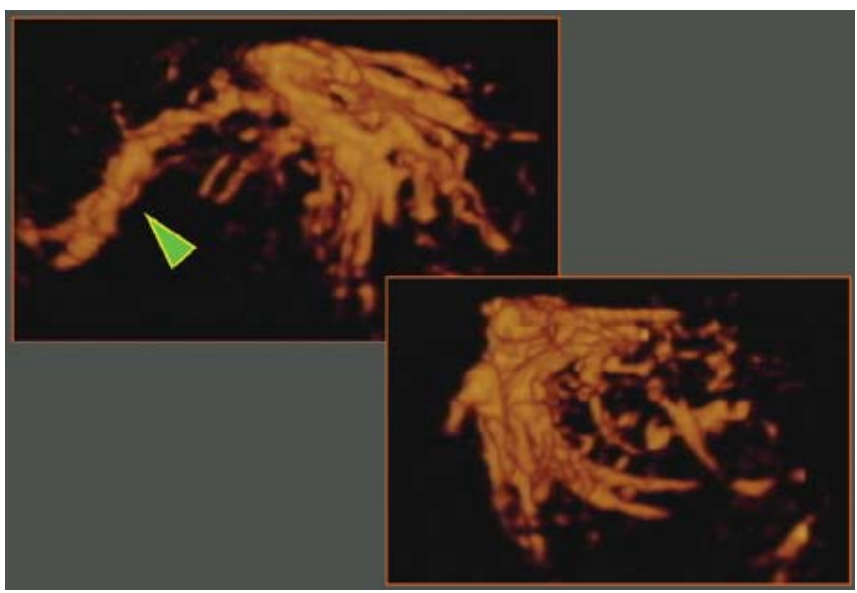

Fig. 27: Three-dimensional power Doppler images of an ovarian carcinoma. Feeding vessels are demonstrated (arrow head)

Three-dimensional images of blood vessels show feeding vessels to an ovarian carcinoma (Fig. 27) and irregular and rich vessel structure in it (Fig. 28). Two-dimensional ultrasound depicts only a part of tumor vascularity (Fig. 29A). However, a 3D Doppler image shows the whole vascularity which sometimes helps to distinguish a tumor of GI tracts from an ovarian tumor (Fig. 29B). Combined evaluations of morphology and neovascularity by $3 \mathrm{D}$ power Doppler ultrasound may improve early detection of ovarian carcinoma. ${ }^{4}$

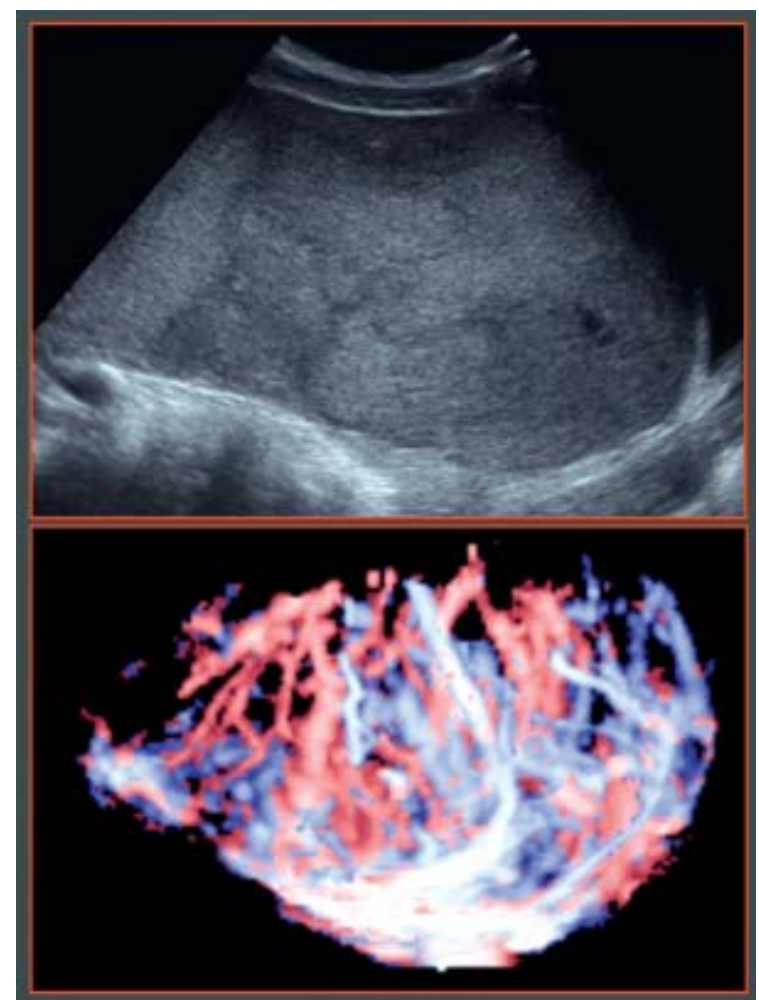

Fig. 28: Dysgerminoma. (Upper) A tomographic image. (Lower) A 3D Doppler image
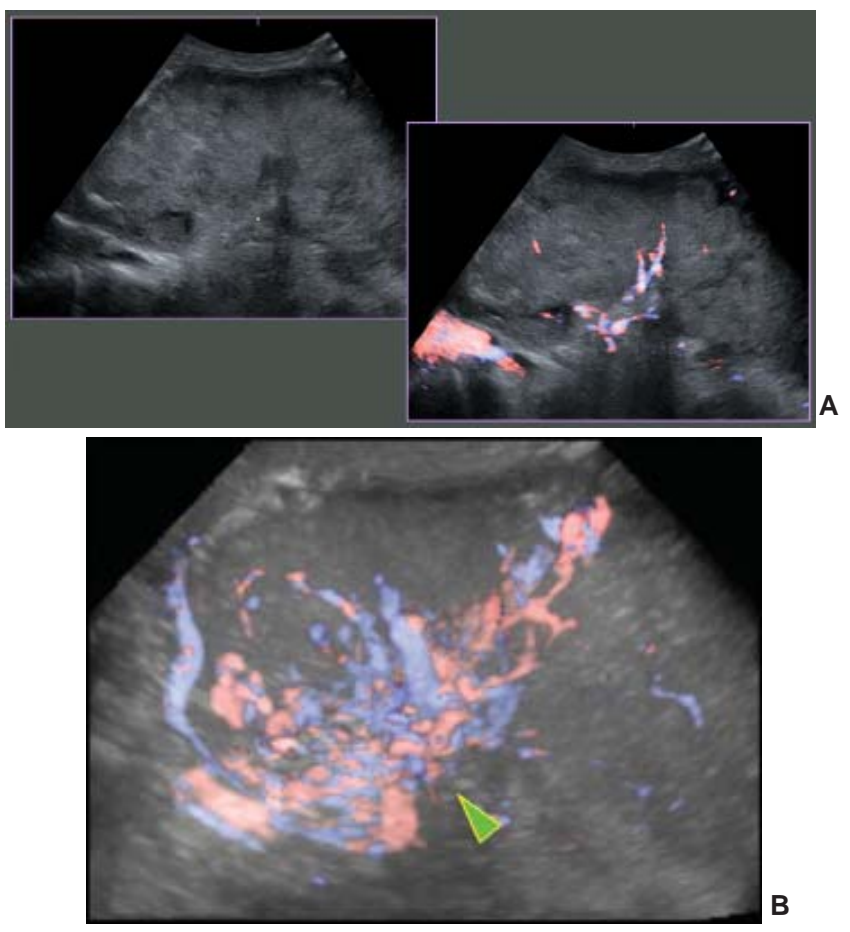

Fig. 29: Adenocarcinoma of the sigmoid colon. (A) A tomographic image and a 2D Doppler image. (B) A 3D Doppler image demonstrates that blood flows concentrate into the middle of the abdomen (arrow head), implying that the tumor is not an ovarian tumor 


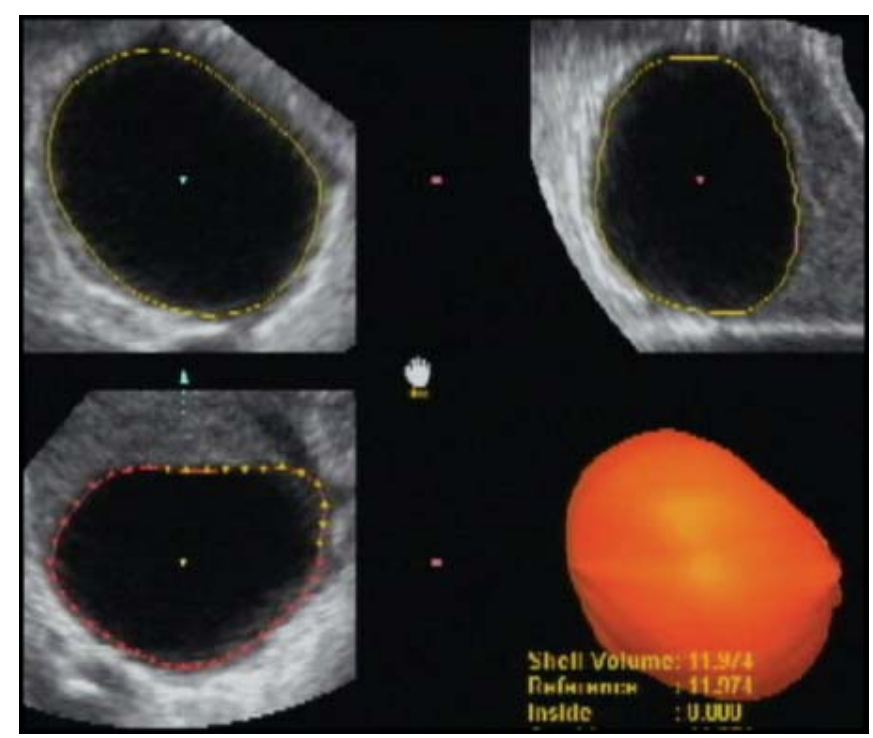

Fig. 30: Automatic extraction of an ovarian cyst and volume measurement

\section{Volume Measurement}

The volume of an ovarian cyst or tumor can be measured accurately with 3D ultrasound. A cyst may be extracted and its volume is measured automatically, when the boundary of the cyst and its surrounding tissue is clear enough (Fig. 30). Even the boundary is not clear enough because of internal echoes, the cyst may be extracted by tracing the boundary manually and the change of its size can be followed up.

Once an ovarian cyst or tumor is extracted, its surface rendered 3D image (Fig. 30 lower right) and transparent 3D image with power Doppler (Fig. 31) can be obtained.

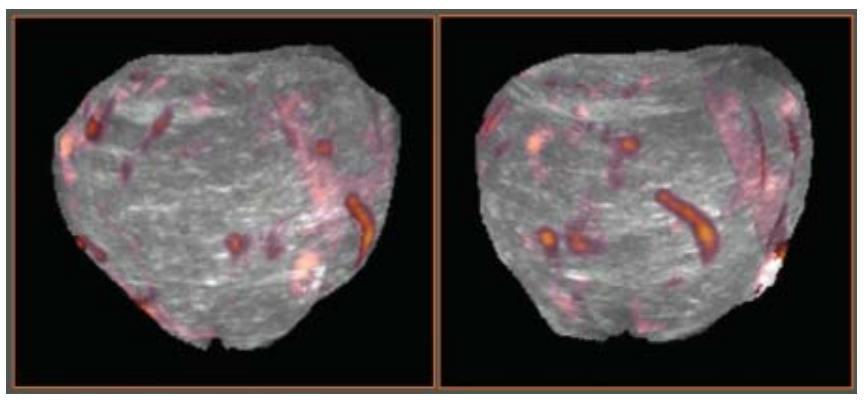

Fig. 31: Transparent 3D images of a hemorrhagic corpus luteum The hemorrhagic corpus luteum was extracted manually and displayed with power Doppler images

\section{CONCLUSIONS}

Three-dimensional ultrasound is useful not only in obstetrics but also in gynecology because of its ability to demonstrate any section and 3D images which cannot be obtained by 2D ultrasound and because of the function of volume measurement.

\section{REFERENCES}

1. Thiel JA, Suchet IB, Lortie K. Confirmation of Essure microinsert tubal coil placement with conventional and volume-contrast imaging three-dimensional ultrasound. Fertil Steril 2005;84:5048.

2. Baba K, Ishihara O, Hayashi N, Saitoh M, Taya J, Kinoshita K. Where does the embryo implant after embryo transfer in humans? Fertil Steril 2000;73:123-5.

3. Baba K, Io Y.3D ultrasound in obstetrics and gynecology. Medical View (Tokyo), 2000.

4. Kupesic S, Plavsic BM. Early ovarian cancer: 3-D power Doppler. Abdom Imaging 2006 Jan 30 [Epub ahead of print]. 
Subscription information:

\section{Annual subscription:}

Individual: Rs. 1600.00

$\$ 130.00$

$£ \quad 75.00$

Institutional: Rs. 3000.00

$\$ 160.00$

$£ \quad 100.00$

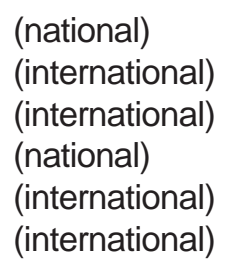

(national)

(international)

(international)

(national)

(international)

(international)

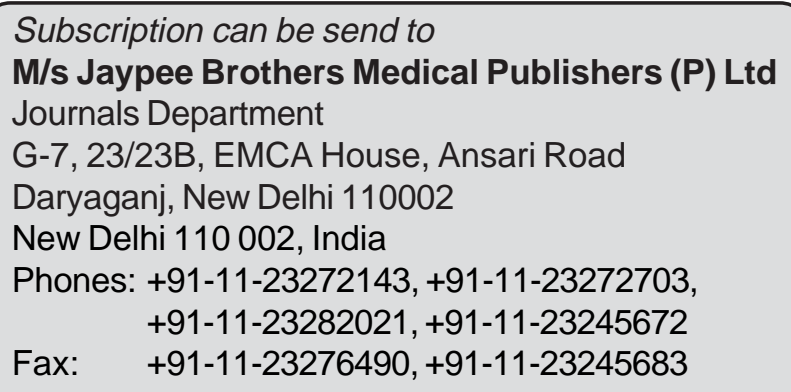

This journal is published quarterly, i.e. January-March, April-June, July-September and October-December, every year. Dollar rates apply to subscribers in all the countries except the UK and the Republic of Ireland where the pound/ sterling price applies. All subscriptions are payable in advance and all the rates include postage. Journals are sent by air to all the countries except Indian subcontinent. Subscriptions are on an annual basis, i.e. from January to December. Payment will be made by sterling cheque, dollar cheque, credit card or directly through our bank account at the following address:
1. Our banker's name
Canara Bank, Netaji Subhash Marg
2. Telephone No:
Darya Ganj, New Delhi 110002
3. Fax No:
011-23273015, 011-23273849
4. Telex Number
011-23255606
5. Our Current A/c. No.
3166291
6. Amount to be transferred
3828 in the name of:
7. Swift code No.
JAYPEE BROTHERS MEDICAL PUBLISHERS (P) LTD., NEW DELHI
CNRB IN BB DFM

For further queries please do not hesitate to contact MR TARUN DUNEJA at the above address.

\section{JAYPEES JOURNAL ADVERTISEMENT RATES} (For the Print Issues)

Page

Back cover-colour Inside front cover-colour Inside back cover-colour Special position*-colour Inside full page-colour

\begin{tabular}{lrl} 
& Single issue & \\
\hline Rs. 25,000 & $\$ 850.00$ & $€ 700$ \\
Rs. 20,000 & $\$ 750.00$ & $€ 500$ \\
Rs. 15,000 & $\$ 650.00$ & $€ 400$ \\
Rs. 12,500 & $\$ 600.00$ & $€ 350$ \\
Rs. 10,000 & $\$ 500.00$ & $€ 300$
\end{tabular}

*First page, page facing editorial board, page facing table of contents. Cover page advertisements not available for a single issue.

\section{Technical Details}

Paper size

Print size

Digital file format

Printed on art paper using offset printing.

\section{Schedule}

Issues are published in the months of January, April, July and October.

$8.5 \times 11.5$ inches

$7 \times 10$ inches

EPS on CD (at 300 dpi resolution) print date.

\section{Payment Details}

- Payment should favour "Jaypee Brothers Medical Publishers Pvt (L) " and should be payable at New Delhi, India

- Payment to be done at the time of submitting the advertisement material/booking the advertisement. Please send your advertisement request, payment and advertisement material to the address given above. Editorial board reserves the right to accept or decline the advertisement. 